\title{
A Mobility Model for Personal Networks (PN)
}

\author{
Yanying Gu, R. Venkatesha Prasad, Ignas Niemegeers \\ Center for Wireless and Personal Communication (CWPC), \\ Delft University of Technology, Delft, The Netherlands \\ Email:\{y.gu, vprasad, I,niemegeers\}@ewi.tudelft.nl
}

\begin{abstract}
Being highly user-centric, Personal Networks (PN) enable interconnection between various devices of a user (personal devices) in different geographic locations, such as home, office, car, etc., to form one secure network for the user. In this paper we analyze properties of node mobility in PNs first. We address typical PN scenarios where personal devices in the vicinity naturally form a small cluster and move in groups to support the demands of a user. Based on the PN mobility properties studied on the basis of the scenarios, a PN Mobility Model (PNMM) is proposed. PNMM can be used to evaluate the techniques and protocols designed for PN implementations. We evaluate PNMM, compare it with other mobility models, and show that PNMM applies better than other models with respect to the behaviors of nodes in PNs. Moreover, to evaluate mobility models some evaluation methods have been proposed to examine to what degree mobility model can represent the properties of a certain scenario. This includes node mobility, heterogeneity, relative node mobility in a group, and dynamics of group merge and split
\end{abstract}

Keywords-Personal Network (PN), ad hoc network, PN Mobility Model

\section{Introduction}

With the aim of supporting the future needs of a person, a Personal Network (PN) [1] [2] - shown in Fig. 1- interconnects all the devices belonging to a person to form a private and secure network. Thus a user can freely and safely use all his/her communicating and computing devices called personal nodes, and access personal or public services through them. All these personal nodes in PNs may be equipped with one or more communication technologies, such as WPAN, WLAN, UMTS networks, etc. And personal devices are separately involved in various networks using different kinds of communication technologies. By integrating all the personal nodes a PN should be designed to minimize the interference and take advantage of the coexistences of these existing technologies. As shown in Fig. 1, a Personal Area Network (PAN) includes all the devices within a range of a few meters around a person, whereas, a PN extends the range of a PAN beyond this boundary giving it a global scope. Personal nodes in different places, such as home, office,

Please use the following formal when citing this chapter:

Gu, Y., Prasad, R. V., Niemegeers, I., 2008, in IFIP International Federation for Information Processing, Volume 265, $\wedge$ dvances in $\Lambda \mathrm{d}$ Hoc Networking, eds. Cuenca, P., Guerrero C., Puigjaner, R., Serra, B., (Boston: Springer), pp. 13-24. 
vehicle, class room, etc., are covered by a PN. Thus personal nodes with different wired or wireless technologies in the same or different places should cooperate with each other to form one network to offer flexible personal services anywhere and at anytime. Some typical scenarios in PNs are explained in the next section to give an overview of the possible behaviors of a PN, which contain the detailed description of PN services and applications.

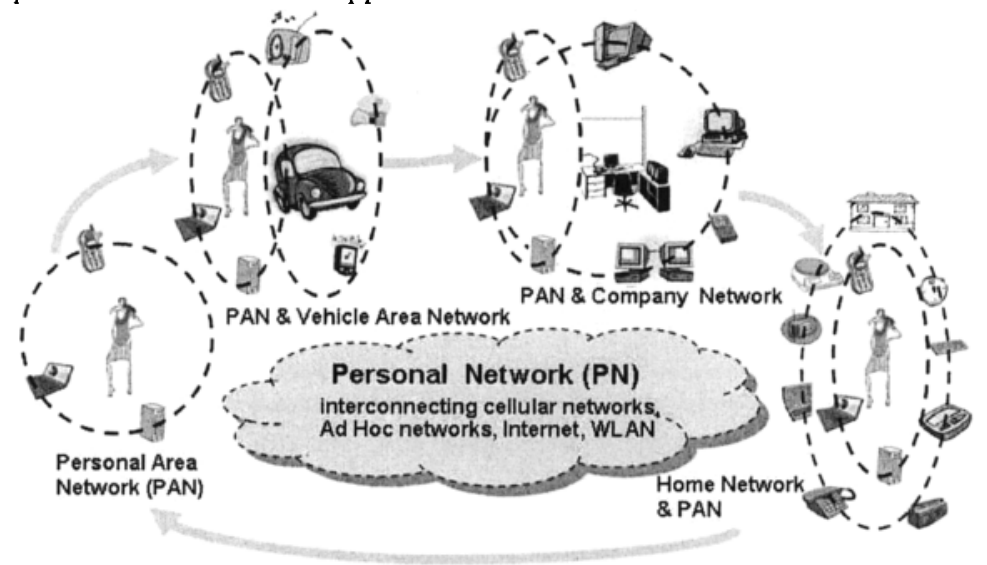

Fig. 1 Personal Network.

Since the actual mobility traces of personal nodes are not available at present, in order to evaluate the techniques and protocols designed for a $\mathrm{PN}$, it is necessary to use a mobility model to describe the behavior of personal nodes in a PN as close to reality as possible. Moreover, different mobility models influence the performance of the designed protocols, and may offer entirely different results. A protocol may work well for a scenario with a particular mobility model; but perform poorly with another mobility model. Thus, a mobility model precisely representing the movement pattern of personal nodes (henceforth simply called as nodes) of a PN can predict whether the proposed techniques and protocols perform well in the future implementations.

Although some of the existing mobility models [3] can offer partial solutions to represent the behavior of mobile nodes in ad hoc networks, little has been done to model the mobility of nodes in a PN setting, especially in user-centric network which is different from simple ad hoc networks already studied in depth. A PN includes personal nodes in various wired and wireless networks, some of the nodes may never move, and others move, which makes the mobility model of a PN complex and different from the usual ad hoc network case studied in the literature [3]. Moreover, nodes naturally move in groups. For example, a person takes his/her mobile phone, PDA, laptop and some sensors on his body together which form a PAN; he/she moves from home to the work place thus these personal nodes move in a group along with the user. Unlike a group mobility model designed for partic- 
ular scenarios, such as column mobility model [3], where a group of soldiers move together with less relative mobility inside a group, because they move along a given line and in one direction, a $\mathrm{PN}$ has a dynamic relative mobility within a group of nodes. For instance, a user may put his/her MP3 player in his/her left or right pocket, which makes a dynamic relative mobility between the MP3 player and his/her other personal devices. The behavior of nodes in a PN is different from the scenarios that have been already modeled [2]. Thus, a new mobility model specifically for PNs is indeed needed for the evaluation of PN protocols [1].

In this paper, we attempt to capture the various mobility aspects of PN nodes. The model evolved here would be useful in studying the PNs and studying its impact, usefulness and the pitfalls of the existing ideas of PN design. The remainder of this paper is organized as follows. In Section 2 we explain the requirements of a PN mobility model which are based on various PN scenarios and use cases. In Section 3 some existing mobility models are introduced and compared with the PN mobility model requirements to show the differences between them. In Section 4 the descriptions of the PN mobility model (PNMM) are highlighted. Section 5 also offers explanations of various evaluation results of the proposed PN mobility model and other existing mobility models. Finally, Section 6 summarizes our work with conclusions including the contributions and performance improvements of PN mobility model.

\section{From PN Scenarios to PN Mobility Model Requirements}

A mobility model for a PN should capture the features of the movement of nodes in a PN. Since PN is a user-centric design to support personal applications in various scenarios, personal services for different persons are to be defined first. In this section, we describe some PN services and applications for various scenarios, and from which we draw a picture of the nodes' mobility in a PN, and get the requirements for PN mobility model. Some of the use cases are as follows:

- A health-monitoring application:

Monitoring the health condition of a disabled or an elderly person is a potential personal service in a PN, which can collect useful data not only for emergency situations, but also for daily health monitoring and maintenance. Thus a PN incorporates sensing and actuating devices linked to a health-monitoring server at home. Some sensing devices are installed in various places at home to collect raw data for health applications. The user takes some wearable devices with him/her, which move together with the user. Thus we can draw the first PN mobility model requirement, which is the heterogeneous mobility nature of nodes: some personal nodes never move, and some can move.

While a person moves from room to room, some wearable and mobile devices moves in a group with him/her for the purpose of monitoring the user and support health applications. Thus we get another PN mobility model requirement: person- 
al devices cooperate to support the demands of a user, they are not independent, and usually move in groups.

- Business environment

Considering the physical environment of a person, e.g., his/her home, office, car, etc., a person has some groups of personal devices in different places. A businessperson has a number of personal devices in different places for his/her social and private needs. While the businessperson, with his/her PANs, moves in these places, the number of personal devices in each place varies. Thus we conclude the third PN mobility model requirement: the number of personal nodes in each group is not fixed. In addition, the coverage areas of these places are also different. For example, the coverage areas of a room and a car are different. When personal nodes move in these places, they may cover the whole area to support the users. Thus the area covered by a group of personal nodes, such as an office, a vehicle, a bedroom, etc., is called group coverage area in this paper. So the fourth PN mobility model requirement is: the coverage area of each group is not fixed, and we can set a maximum value.

As the businessperson leaves his/her office and enters his/her car. Some personal devices are left in the office; other personal devices are carried by the person who moves into the coverage area of the on-broad car network. So the fifth PN mobility model requirement is: group of nodes split. When the person moves to his/her car, while he/she is making a conference call using his/her PDA, then the $\mathrm{PN}$ can enable the conference call seamlessly handing over to the on-broad car network, where on-board speakers, microphones and the PDA are used together to offer the conference call service. Thus the group of devices around the person and the group of personal devices in his/her car merge. The sixth PN mobility model requirement is: two groups of personal nodes can merge into one group.

- A remote babysitting application:

In the case of a mother visiting a friend's house her sleeping baby should be tracked. A PN can be designed for her need, thus she can divide her personal nodes into various groups according to the demands. For this application the mother can decide her nodes into two groups: one group is in the room monitoring her baby; and the other group of nodes carried by her to display the tracking data of her baby. Thus we get the seventh PN mobility model requirement: the number of groups of personal nodes can be pre-set. When the mother moves from her house to her friend's house by her car, the velocity of the group of personal devices in the car is dynamic and limited within a maximum value, because the vehicle velocity in a city is limited. So the eighth PN mobility model requirement is: the group velocity is same for the member nodes, and can be limited by a maximum value. After an hour, the mother comes back to home, and her baby is awake. At home, the two groups merge into one. The mother does housework and moves between different rooms; at the same time, she needs to keep her eyes on her baby and uses the baby monitoring service. Thus, the nodes carried by the mother and the devices near the baby should work together to support the baby monitoring 
service. And the devices move together with the mother have relative mobility with respect to other devices near the baby. Thus we can conclude the last PN mobility model requirement: the personal devices included in the same group can have relative mobility with each other.

\section{Table 1 PN Mobility Requirements.}

Requirements

1 The mobility heterogeneity of personal nodes: some personal nodes never move, some can move

2 Personal devices cooperate to support the demands of a user, they are not independent, and usually move in groups.

3 The number of personal nodes in each group is not fixed.

4 The coverage area of each group is not fixed, and we can set a maximum value.

5 Group of nodes split.

6 Two groups of personal nodes can merge into one group.

7 The number of groups of personal nodes can be pre-set.

8 The group velocity is same for the member nodes, and can be limited by a maximum value.

9 The personal devices included in the same group can have relative mobility with each other.

Based on these examples of user scenarios, we conclude nine PN mobility model requirements, which are listed in the Table 1.

\section{Related Work}

In this section, we introduce some existing mobility models, and examine whether they can be used to model mobility in PNs.

- Random walk mobility model [4]:

Random walk mobility model is a simple mobility model developed to present the wandering movement of nodes. In this model, a mobile node randomly chooses a direction $\theta$ in the range $[0,2 \pi]$ and a speed in a pre-defined range $\left[V_{\min }, V_{\max }\right]$. A mobile node chooses a new direction and a new speed after traveling constantly for a time $t$.

- Random waypoint mobility model [5]:

Random waypoint mobility model is the most commonly used by researchers for simulating ad hoc networks with mobility. In this model, a mobile node travels towards a randomly chosen destination with randomly chosen speed in the range of $\left[V_{\min }, V_{\max }\right]$. After the node reaching the destination, it stops for a duration, and repeat the process again.

- Random direction mobility model [6]: 
Similar to the random walk mobility model, a mobile node in this model chooses a random angular direction and a random speed. The difference is that a mobile node needs to travel to the edge of the simulation area, and then it can change direction and speed. The above three mobility models assume all the nodes are independent. Thus these mobility models do not meet most of the PN mobility requirements.

- Reference point group mobility model (RPGM) [7]:

Reference point group mobility model gives a solution to modeling group mobility. In this model, the movement of a group is represented by the logical center of the group moving along a series of pre-defined check points. However, this model assumes a group of nodes always move together, which does not meet the PN mobility model requirement 5 and 6 .

- Reference region group mobility model (RRGM) [8]:

Reference region group mobility model defines a reference region as a destination to which a group of mobile nodes move. If a mobile node arrives at the reference region, it waits for the other mobile nodes to arrive. However, PN scenarios explained in the last section are different from the scenarios considered in RRGM. A group of personal nodes in PNs should have to move together to satisfy the need of a user at the same time. Thus the RRGM is not suitable for PNs.

Different mobility models typically focus on a specific scenario. Although some mobility models can meet part of the PN mobility requirements, none of them meets all the requirements. Thus a new mobility model for PNs is needed.

\section{PN Mobility Model}

A PN mobility model (PNMM) is explained in the following steps, and principles to meet each PN mobility model requirement (R1-R9) are highlighted.

\section{- Step 1: Initialization}

In order to meet R1, there are some non-moving nodes, and their positions are chosen randomly in the simulation area. To meet R3, each mobile node randomly selects one of $M$ groups, where $M$ is the mean value of the maximum and minimum number of groups ( $X$ and $Y$ ). In fact, setting a minimum and maximum limit is for practical reasons. That is PNs have limited number of devices and usually they have at least 1 to 3 groups (Home, office and car group). Each group covers a circular region with the center chosen randomly in the simulation area and a radius chosen randomly in the range of $\left[0, R_{\max }\right]$, which satisfies $\mathrm{R} 4$. And the location of a node is chosen randomly in its group coverage area.

- Step 2: Movement of a group 
In each time interval $\alpha$, a group destination area is selected, to which a group moves. The center of the destination area is chosen randomly, which can be reached within a maximum value $V_{g}$ meeting $\mathrm{R} 8$. The radius of the destination area is selected randomly and satisfies the rules explained in Step 1. For each node in the group, it randomly selects a point in the group destination area, and then moves towards it with a constant velocity. Thus all the nodes in a group move together at the same time, which meets R3. Moreover, the nodes in the same group have relative random mobility, because their position is re-chosen randomly inside the group coverage area in each time interval $\alpha$, which meets R9.

\section{- Step 3: Group merging}

To meet R6, group merging is planned in each time interval $c \times \alpha$. When $c=1$, the group merge interval is as the same as the group movement interval $\alpha$. If two groups can reach each other within the maximum velocity $V_{\mathrm{g}}$ in a time interval $\alpha$, they can merge into one group with the center randomly chosen in the area, where these two groups can arrive. To meet R7, the methods controlling the number of groups bigger than $Y$ are given: if $n_{m p}{ }^{(t)}$ pairs of groups can be merged, the maximum number of merged group pairs is $m$, where $m=\operatorname{Min}\left(n_{m p}^{(t)},\left(n_{z}^{(i)}-\gamma\right)\right)$, and $n_{g}{ }^{(i)}$ is the group number. Thus the number of merged group pairs is chosen randomly in the range of $[0, m]$.

\section{- Step 4: Group division}

To meet R5, the group division is considered in every time interval $d \times \alpha$. When $d=1$, the group merge interval is as the same as the group movement interval $\alpha$. A group can be divided into two groups. The destination areas of the two groups should be chosen in the same way as described in Step 2. Each node in the original group can randomly choose one of the two groups. To control the number of groups smaller than $X$ and meet $\mathrm{R} 7$, the number of divided groups is randomly chosen in the range $\left[0,\left(X-n_{g}^{(t)}\right)\right]$.

- Step 5: Group merging and dividing at the same time

For a group chosen to be both merged and divided, the division is considered first. Some nodes chosen randomly move to another group (following Step 4). Other nodes undergo merging (following Step 3).

\section{Evaluation}

In this section, we evaluate mobility models with respect to the requirements of PNs. We divide the nine PN mobility model requirements into four categories, which describe different properties of node mobility in PNs: (1) the heterogeneity of node mobility (Requirement 1 ); (2) group mobility \& node mobility inside a group (Requirements $2,4,8,9$ ); (3) group merging \& dividing (Requirements 5 , 
6); (4) group numbers \& number of nodes in each group (Requirements 3, 7). For each category, we discuss and compare the existing mobility models and our PNMM.

- The heterogeneity of node mobility:

Because nodes are equipped with different technologies, including different types of networks, and used for different kinds of applications for a user, heterogeneity is a key property in PNs. Personal nodes have heterogeneous mobility types as described in Section 2. In order to evaluate the heterogeneity of node mobility, we define a Mobility Heterogeneity Value (MHV). Assume all the nodes considered have $n$ levels of mobility; in each level, nodes have the same mean velocity $v$; the percentage of number of nodes in each level is $q$. Thus the mean velocity of all the nodes, $\bar{V}$, is

$$
\bar{V}=\frac{1}{n} \sum_{i=1}^{n} v_{i} q_{i}
$$

And Mobility Heterogeneity Value (MHV) is

$$
M H V=\sum_{i=1}^{n}\left[\left(\bar{V}-v_{i}\right)^{2} \times q_{i}\right]
$$

A higher value of $M H V$ means high node mobility heterogeneity.

For all the existing mobility models introduced in Section 3, their $M H V$ are zero, because every node has the same maximum velocity; there is no velocity difference among the nodes. For our proposed PN mobility model, we separate all the personal nodes into two kinds: 'never move', $n_{s}$, and 'can move', $n_{m}$. Thus the $\bar{V}$ and $M H V$ of the (PNMM) is

$$
\bar{V}_{P N M M}=\frac{1}{2} \times\left(\frac{V_{g}}{2}\right) \times\left(\frac{n_{m}}{N}\right)
$$

$$
M H V_{P N M M}=\left(\bar{V}_{P N M M}-\frac{V_{g}}{2}\right)^{2} \times\left(\frac{n_{m}}{N}\right)
$$

Thus the node mobility heterogeneity value (MHV) of PNMM is higher than any other mobility model.

- Group mobility and node mobility inside a group: 
Based on the PN mobility model requirement $2,4,8,9$, the mobility of groups of personal nodes and the mobility of nodes in each group should be represented. For the mobility of groups, in reference point group mobility model (RPGM), each group can freely move inside the considered area. However, in reference region group mobility model (RRGM), a group has a pre-specified destination area then the group moves to the destination along a curved route, because RRGM models search or rescue scenarios with groups of persons. In our proposed PN mobility model, random movement of groups can be modeled by randomly choosing the destination area of each group at every time interval $\alpha$.

To meet the PN mobility model Requirement 9 , nodes inside a group should have relative mobility. We define a Relative Mobility in Group ( $R M G), \delta$ to describe the relative mobility level of personal nodes in a moving group. Firstly, the Average Possible Coverage Area of a Node (APCAN) of node $j, \kappa_{j}^{N}$ is defined as the total area, in which a node in a group may possibly reach, at any moment during the simulation time period. We define the Average Possible Coverage Area of a Group $(A P C A G), \kappa^{G}$ as the total possible reachable area, in which all the nodes in a group move at any moment during the simulation time period. Assume $n$ nodes are considered in a group then $R M G$ is defined as

$$
\delta=\left(\frac{1}{n \times \kappa^{G}}\right) \times \sum_{i=1}^{n} \kappa_{j}^{N}
$$

With a bigger $A P C A N$ of every node in a group with respect to the $A P C A G$, the relative mobility of nodes in that group is higher.

To compare the performance of relative mobility of nodes in a group, we calculate $R M G, \delta$ of RPGM, RRGM and our PNMM. We assume one group of $n$ nodes, with the maximum group velocity $V_{g}$. In RPGM [7], there are $n$ reference points (RP) for every node in a group. Each node can move randomly in the circular area with a specified radius with respect to its $\mathrm{RP}$. Thus $\kappa_{j}^{N} \leq \kappa^{G}$, only then all the RPs of $n$ nodes is the same, $\kappa_{j}^{N}=\kappa^{G}$ thus $\delta_{R P G M} \leq 1$. In RRGM [8] we assume the destination area of a group is $\kappa^{G d}$ and $\kappa_{j}^{N}=\kappa^{G d}$. However, $A P C A G$ of RRGM is bigger than the destination area of a group, $\kappa^{G} \geq \kappa^{G d}$, because each node in the group moves with a different velocity, when one node with a higher velocity arrives at the group destination area first, while another node with a lower velocity may still be moving towards the destination area. Thus $\delta_{R R G M} \leq 1$. In PNMM, since a node can randomly choose its location in the destination area of the group, $\kappa_{j}^{N}=\kappa^{G}$, and $\delta_{P N M M}=1$. PNMM has the highest relative node mobility inside a group. 
- Group merging \& division

As explained in Section 2, in some PN scenarios, a group of personal nodes are divided into two groups, and two groups of personal nodes merge into one group. There are only two mobility models that can present the behavior of group merging and division, which are RRGM and our proposed PNMM. In RRGM, two groups can merge, if they meet two requirements: (1) they are small groups with a few nodes in each group; (2) the groups have paused at the destination for a predefined period of time. These two requirements are not suitable for the PN scenarios. Thus the cluster merge rules of RRGM can not be used in PNs.

In PNMM, group merging and division is performed randomly amongst all the groups. To show the dynamics of group merging and division from the view of each individual node, we define Node Change Rate (NCR) as the rate of number of nodes that join or leave a group. If the total simulation time is $T$, the time interval to plan group merge and division is $\alpha$ (we consider $c=d=1$ in the evaluation part), there are $m$ time slots considered, where $m=T / \alpha$, and $n_{m d, i}$ represents the number of nodes that join or leave a group in the $i^{\text {ih }}$ time interval.

$$
N C R=\frac{\sum_{i=1}^{m}\left(n_{m d, i} / \alpha\right)}{m}
$$

Based on the rules of group merging and division as described in Section 4, the NCR of PNMM is,

$$
N C R_{P N M M}=\frac{3 N}{2}\left(\frac{X-Y}{X+Y}\right)
$$

\section{- Group numbers \& number of nodes in each group}

Since a PN belongs to a user and carry out the demands of the user, the user can organize his/her devices in a number of groups for his/her convenience. The number of groups can be decided by the user, and these groups can merge and separate, which results in the number of groups varying in the range of $[Y, X]$. Based on the rules of determining the number of pairs of groups to be merged, $n_{m}{ }^{(1)}$, and the number of group pairs to be divided, $n_{d}{ }^{(t)}$, the number of groups in PNMM is controlled and is in the range of $[Y, X]$. However, none of the other mobility model can give a solution of controlling the number of groups.

For the number of nodes in each group, in RPGM and RRGM, it should be prespecified for each group. In PNMM, a node can randomly choose a group, thus for each case, the number of nodes in each group in a $\mathrm{PN}$ is different, which show the 
heterogeneity of the sizes of groups in PN and is suitable for PN scenarios explained in Section 2.

Based on the above evaluation, PNMM proves to be the best fit for R1-R9. However, in the current literature, ad hoc mobility models have been used in the evaluation of various protocols. The mobility in ad hoc networks offer an independent and random movement for each mobile node, while the mobility in PNs focuses mainly the heterogeneity in node movement and random group mobility. Because of these unique PN mobility properties, which are different from ad hoc networks, protocols and techniques proposed for PNs should be evaluated by PNMM to examine whether they work well in PNs.

\section{Conclusion and Future Work}

To model the node mobility in PN, the behaviors of the personal nodes are analyzed, and the desirable properties of PN node mobility are summarized as PN mobility model requirements in this paper. Based on nine PN mobility model requirements, PNMM is designed: it has high heterogeneity of node mobility; it enables group mobility and dynamic node mobility inside a group; it models group merging and division; it can control the number of groups; and it organizes groups with different number of nodes in each group. Another contribution in this paper is that we propose some mobility modeling evaluation methods: a Mobility Heterogeneity Value (HMV) for comparing the heterogeneity of node mobility, a Relative Mobility in Group ( $R M G$ ) to present the relative mobility level of nodes in a moving group, and a Node Change Rate (NCR) to show the dynamics of group merging and division from the view of each individual node. These evaluation methods give a way of evaluating mobility models to test how realistic they can describe the mobility properties in the real situations.

The proposed PNMM can be used to evaluate the protocols and techniques designed for PNs, such as self-organization protocols [9], clustering protocols [10], context awareness [11], service discovery and management [12], network mobility (NEMO) [13] management protocols, etc. These protocols and techniques can be tested by using our PNMM to examine whether they can work well in PNs. Moreover, by considering the unique properties of mobility in PNs, the protocols and techniques designed for PNs should be equipped with some special mechanisms to fit the PN scenarios.

Additional work must still be done to deeply analyze the influence of the environments on the mobility for PNs, such as collision avoidance, congestion avoidance [14], etc. Moreover, PNs contains devices involved in different types of networks, such as UMTS, ad hoc networks, sensor networks [15], etc. The particular behaviors of personal devices in these networks should be considered in the future work of mobility modeling for PNs. For example, swarm behaviors [16] of devices in mobile sensor networks should be investigated to address the details of personal nodes mobility in PNs. 


\section{Acknowledgment}

This work is partially funded by the EU IST MAGNET Beyond Project and the Dutch Freeband PNP2008 Project.

\section{References}

[1] Niemegeers, I., \& Heemstra de Groot, S., (2003). Research issues in ad-hoc distributed personal networking. Wireless Personal Communications, 26(2), 149-167.

[2] Lu, L., et al. (2005). D1.2 Initial Architecture of Personal Networks. IOP GenCom Project QoS for Personal Network at Home Deliverable.

[3] Camp, T., Boleng, J., Davies, V., (2002). A Survey of Mobility Models for Ad Hoc Network Research. Wireless Communication \& Mobile Computing (WCMC), 2(5), 483-502.

[4] Sanchez, M., Manzoni, P., (2001). A java based simulator for ad-hoc network. Future Generation Computer Systems, 573-583.

[5] Johnson, D., Maltz, D., (1996). Dynamic source routing in ad hoc networks. Mobile Computing, 153-181.

[6] Royer, E., Melliar-Smith, P.M., Moser, L., (2001). An analysis of the optimum node density for ad hoc mobile networks. IEEE International Conference on Communications.

[7] Hong, X., Gerla, M., Chiang, C., (1999). A group mobility model for ad hoc wireless networks. ACM International Workshop on Modeling and Simulation of Wireless and Mobile Systems (MSWIM).

[8] Ng, J.M., Zhang, Y., (2005). Reference Region Group Mobility Model for Ad Hoc Networks. Wireless and Optical Communications Networks (WOCN).

[9] Lu, W., Gu, Y., Prasad, R.V., Lo, A., Niemegeers, I., (2007). A Self-organized Personal Network Architecture. $3^{\text {rd }}$ International Conference on Networking and Services (ICNS'07).

[10] Gu, Y., Lu, W., Prasad, R.V., Lo, A., Niemegeers, I., (2007). Clustering for Ad Hoc Personal Network Formation. International Conference on Computational Science (ICCS).

[11] Sanchez, L., Lanza, J., Olsen, R., Bauer, M., Girod-Genet, M., (2005). A Generic Context Management Framework for Personal Networking Environments. $3^{\text {rd }}$ Annual International Conference on Mobile and Ubiquitous Systems.

[12] Stephen, H., \& Aruna, S., (2006). Service Composition for Mobile Personal Networks. $3^{\text {rd }}$ Annual International Conference on Mobile and Ubiquitous Systems.

[13] Devarapalli, V., Wakikawa, R., Petrescu, A., Thubert, P., (2005). RFC 3963: Network Mobility (NEMO) Basic Support Protocol. IETF, NEMO Working Group, January.

[14] Williams, S., \& Huang, D., (2006). A group force mobility model. $9^{\text {th }}$ Communications and Networking Simulation Symposium.

[15] Ren, H., \& Meng, H., (2006). Understanding the Mobility Model of Wireless Body Sensor Networks. IEEE International Conference on Information Acquisition.

[16] Anthony, B., Lamont, G.B., (2002). A particle swarm model for swarm-based networked sensor systems. ACM Symposium on Applied Computing. 